\title{
Nanoelectromechanical Heat Engine Based on Electron-Electron Interaction
}

\author{
A. Vikström, ${ }^{1, *}$ A. M. Eriksson, ${ }^{1}$ S. I. Kulinich, ${ }^{2}$ and L. Y. Gorelik ${ }^{1}$ \\ ${ }^{1}$ Department of Physics, Chalmers University of Technology, Kemigården 1, SE-412 96 Göteborg, Sweden \\ ${ }^{2}$ B. Verkin Institute for Low Temperature Physics and Engineering of the National Academy of Sciences of Ukraine, \\ Prospect Nauky 47, Kharkov 61103, Ukraine
}

(Received 12 May 2016; revised manuscript received 3 October 2016; published 9 December 2016)

\begin{abstract}
We theoretically show that a nanoelectromechanical system can be mechanically actuated by a heat flow through it via an electron-electron interaction. In contrast to most known actuation mechanisms in similar systems, this new mechanism does not involve an electronic current nor external ac fields. Instead, the mechanism relies on deflection-dependent tunneling rates and a heat flow which is mediated by an electronelectron interaction while an electronic current through the device is prohibited by, for instance, a spin-valve effect. Therefore, the system resembles a nanoelectromechanical heat engine. We derive a criterion for the mechanical instability and estimate the amplitude of the resulting self-sustained oscillations. Estimations show that the suggested phenomenon can be studied using available experimental techniques.
\end{abstract}

DOI: 10.1103/PhysRevLett.117.247701

In nanoelectromechanical systems (NEMS), the motion of a mechanical object can be significantly affected by single-electron phenomena [1-6]. In particular, it has been shown that such phenomena can be utilized to achieve ground state cooling of the mechanical motion [7-9]. On the other hand, these phenomena also allow for the actuation of the mechanical motion [10]. The study of actuated motion offers possibilities to investigate the multifaceted nonlinear dynamics of NEMS [11-13].

Many mechanisms of the actuation of mechanical vibration have been suggested. The most straightforward method is to pump energy into the mechanical subsystem using an external ac field with a frequency close to that of the mechanical vibration $[1,10,14-16]$. However, it is also possible to achieve an actuation without resorting to direct resonance, for which there are two distinct approaches. The first one is to use an external off-resonant high-frequency field to generate sideband transitions in the system [17], a technique often used in optomechanics [18]. The second approach is to utilize a directed electron flow through the mechanical object, generated by either a bias voltage $[8,19-$ 22] or a temperature drop [9] between two electronic reservoirs. This approach relies on the fact that mechanical motion induces variations in the average charge (or spin) on the mechanical object. These variations generate an electromechanical feedback force which pumps the mechanical motion. However, if the electron flow is blocked, mechanisms relying on charge or spin current no longer work.

In this Letter, we propose a heat-flow-based actuation mechanism in a system where the charge current is blocked. The heat flow is enabled by the coupling of two thermal reservoirs via an electron-electron interaction. Furthermore, the proposed mechanism actuates vibrations despite the fact that the mechanical motion does not affect the average charge on the mechanical oscillator. This is interesting, since, naively, one would think that in this case there can be no electromechanical feedback. However, we will show that such feedback still exists and results in a conversion from thermal to mechanical energy. Therefore, the system could be seen as a nanoelectromechanical heat engine. We present the actuation mechanism by considering a prototype system which well illustrates the phenomenon and which has a structure analogous to existing devices [23].

The prototype system (Fig. 1) consists of a carbon nanotube (CNT) suspended between two electrode leads above a gate electrode. The ends of the CNT are clamped, and it is connected to the leads through high-resistance tunnel barriers. A tip electrode is positioned above the suspended part of the CNT, so that electrons can tunnel also between the tip and the CNT. The CNT is free to oscillate vertically. As the CNT is mechanically deflected toward the

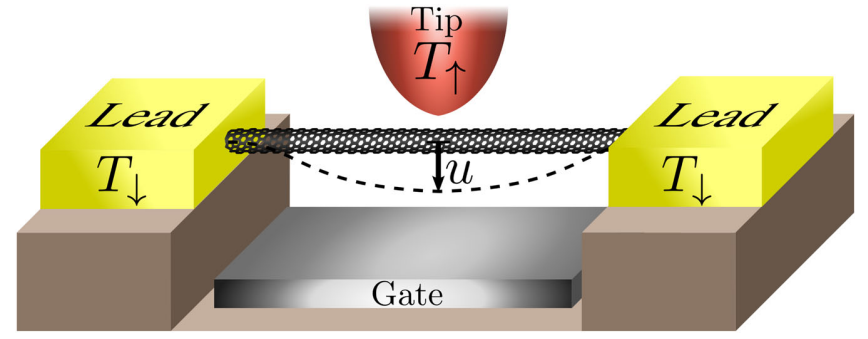

FIG. 1. The prototype system. A CNT is suspended between two leads, below a tip electrode and above a gate. The pair of leads and the tip are two completely spin-polarized electron reservoirs with temperatures $T_{\uparrow}$ and $T_{\downarrow}$ (index according to spin polarization). Electrons can tunnel between the CNT and the reservoirs, but an electron exchange between the two reservoirs is prevented by spin blockade. However, electron-electron interaction couples the two reservoirs, allowing for a heat flow. The tunneling between the tip and the CNT is affected by $u$, the mechanical deflection of the CNT (black arrow), and $u$ is in turn affected by a capacitive force due to the interaction between the charge on the CNT and the gate. 
tip, the rate of electron tunneling between them increases exponentially. A charge on the CNT results in a capacitive force toward the gate electrode. In order to prevent a charge current from the tip via the CNT to the leads, we take the tip and leads to be fully spin-polarized half-metals [24] with spin up $(\uparrow)$ and spin down $(\downarrow)$, respectively; see Fig. 1. The temperature of the two leads is assumed to be set to $T_{\downarrow}$, while the temperature of the tip is $T_{\uparrow}$. Without a loss of generality, we treat the two leads as a single spin- $\downarrow$ reservoir and the tip as a spin- $\uparrow$ reservoir. We also assume that electrons do not change spin during tunneling events. Charge transfer between the two oppositely spin-polarized reservoirs is then blocked, and the two spin subsystems would be decoupled if not for the electron-electron interaction. As we will show, the interaction enables a heat flow between the reservoirs when their temperatures are different: $T_{\uparrow} \neq T_{\downarrow}$.

We will model the CNT as an electronic four-state quantum dot (QD). This is motivated by the assumption that the CNT is short enough for the spatial quantization energy to be larger than all considered energies. The four QD states are the charge-neutral state $|0\rangle$, the two singlepopulated states corresponding to different spin, $|\uparrow\rangle$ and $|\downarrow\rangle$, and the double-populated state $|2\rangle$. These states are characterized by their energies, counted from the chemical potential in the reservoirs $\left(\mu_{\uparrow}=\mu_{\downarrow}\right)$. The neutral state energy is, by definition, $E_{0}=0$. For the single-populated states, $E_{1}^{\sigma}(u)=\mathcal{E}_{1}^{\sigma}+e^{2} / C(u), \sigma=\uparrow, \downarrow$, where $\mathcal{E}_{1}^{\sigma}$ is set by the tuning of the energy levels in the QD relative to the chemical potential and $C(u)$ is the QD-gate capacitance. The double-populated state energy is $E_{2}(u)=E_{1}^{\uparrow}(u)+$ $E_{1}^{\downarrow}(u)+U(u)$, where $U(u)=2 e^{2} / C(u)$ is the electronelectron interaction energy.

To understand the nature of the electromechanical feedback, let us first assume that the CNT is static and consider the electronic dynamics. We characterize the state of the electronic system by the four probabilities of finding the QD in each of its four QD states. These probabilities are governed by the probability flows $w_{\alpha}^{\sigma}(\sigma=\uparrow, \downarrow, \alpha=1,2)$ between different QD states due to tunneling of electrons between the QD and the reservoirs (Fig. 2). In the stationary state, the probability flows are balanced: $w_{\alpha}^{\sigma}=w$. As a consequence, there will be a constant counterclockwise flow of probability in Fig. 2 accompanied by a heat transfer from the hot to the cold reservoir. The heat transfer can be visualized by the following steps: (I) a "cold" spin- $\downarrow$ electron tunnels to the empty QD, (II) a "hot" spin- $\uparrow$ electron with enough energy to overcome the electron-electron interaction energy $U$ tunnels to the QD, (III) the spin- $\downarrow$ electron then tunnels back to the cold reservoir, carrying with it the excess energy $U$, and (IV) the spin- $\uparrow$ electron returns to its reservoir with lower energy, leaving the QD empty, and a new cycle can begin. This heat flow is entirely mediated by the electron-electron interaction and does not involve any exchange of electrons between the reservoirs. In the stationary regime, the heat flow

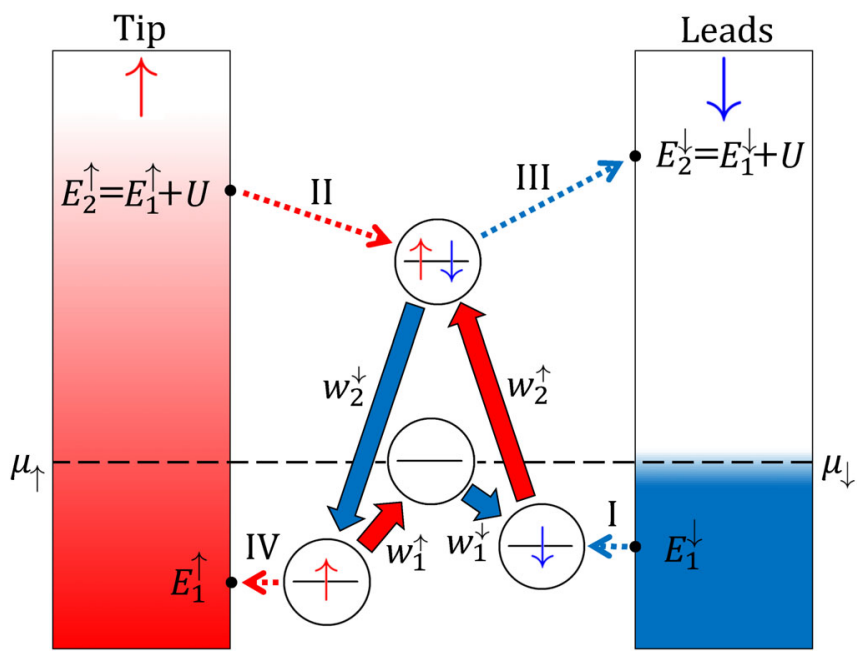

FIG. 2. Schematic energy diagram of the electronic states of the QD and the tunneling processes (dotted arrows). The average flows of probability between the different states are illustrated by the solid arrows $w_{\alpha}^{\sigma}$. The average flow is counterclockwise, since only electrons from the hot electron have enough energy to overcome the electron-electron interaction energy and bring the QD to the double-populated state.

$W$ is proportional to the flow of probability $w$ and energy of the electron-electron interaction $U, W=U w$. Now suppose that we perturb the stationary state by slightly increasing the tunneling rate between the hot reservoir and the QD. This increases the two probability flows $w_{\alpha}^{\uparrow}$. The unaffected exchange of spin- $\downarrow$ electrons with the cold reservoir then results in bottlenecks in the cycle (Fig. 2). The bottleneck effect accumulates probability in the double-populated and neutral states while draining the single-populated states by the same amount. Therefore, the average charge on the QD is unchanged; the increased tunneling of spin- $\uparrow$ electrons to the QD is compensated by the increased tunneling from it. However, the higher probability of double population still increases the capacitive force. The rate of change of the probabilities will be proportional to the probability flow $w$, and thus to the associated heat flow $W=U w$, because of the exponential tunneling dependence on the deflection. The feedback mechanism leading to the electromechanical instability can therefore be understood in the following way. The CNT performs a slight deflection away from its static position toward the tip reservoir (Fig. 1). This will mainly affect the electronic dynamics by increasing the spin- $\uparrow$ tunneling rate due to its strong exponential dependence on the deflection. The bottleneck effect will start to increase the probability of double population and thus the capacitive force toward the gate. However, the electronic response is not instantaneous. Hence, vibrations of the CNT will result in a delayed force which will stiffen and pump the mechanical motion.

The feedback force can be controlled by the temperature difference between the reservoirs. If we swap the temperatures of the two reservoirs, the probability flow in Fig. 2 
would be reversed. The bottleneck effect would then instead drain the double-populated state when the CNT moves closer to the tip. The feedback force would therefore result in softening and damping of the mechanical motion. Furthermore, increasing the temperature difference increases the heat flow and thus the probability flow between the QD states. This enhances the bottleneck effect, generating a stronger feedback. Therefore, both the direction and the strength of the feedback force can be controlled by adjusting the temperatures of the reservoirs.

In order to mathematically model the system, we assume that the dynamics of the CNT is completely characterized by the amplitude of its fundamental bending mode, i.e., the CNT deflection $u(t)$; see Fig. 1 . We describe the time evolution of $u(t)$ as a forced harmonic oscillation:

$$
\begin{gathered}
\partial_{t}^{2} u+\frac{\omega}{Q} \partial_{t} u+\omega^{2} u=\frac{1}{m_{\mathrm{eff}}} F_{\mathrm{el}}, \\
F_{\mathrm{el}}=\operatorname{Tr}\left\{\hat{\rho}\left(-\hat{n}_{\uparrow} \partial_{u} E_{1}^{\uparrow}-\hat{n}_{\downarrow} \partial_{u} E_{1}^{\downarrow}-\hat{n}_{\uparrow} \hat{n}_{\downarrow} \partial_{u} U\right)\right\},
\end{gathered}
$$

with mechanical frequency $\omega$, quality factor $Q$, and effective mass $m_{\text {eff }}$. The force $F_{\text {el }}$ is an average force induced by the electronic subsystem, described by the electronic density operator $\hat{\rho}$. Here, $\hat{n}_{\sigma}=\hat{d}_{\sigma}^{\dagger} \hat{d}_{\sigma}$ is the number operator for spin- $\sigma$ electrons on the QD, where $\hat{d}_{\sigma}$ and $\hat{d}_{\sigma}^{\dagger}$ are the corresponding fermionic annihilation and creation operators, respectively, and, hence, $|\sigma\rangle=\hat{d}_{\sigma}^{\dagger}|0\rangle$ and $|2\rangle=\hat{d}_{\uparrow}^{\dagger} \hat{d}_{\downarrow}^{\dagger}|0\rangle$. We linearize the electronic energies in the mechanical deflection $u$, motivated by it being small compared to the distance to the gate. The dynamics of the electronic subsystem obeys the quantum Liouville equation

$$
\begin{aligned}
i \hbar \partial_{t} \hat{\rho} & =\left[\hat{H}_{\mathrm{QD}}+\hat{H}_{R}+\hat{H}_{T}, \hat{\rho}\right], \\
\hat{H}_{\mathrm{QD}} & =E_{1}^{\uparrow}(u) \hat{n}_{\uparrow}+E_{1}^{\downarrow}(u) \hat{n}_{\downarrow}+U(u) \hat{n}_{\uparrow} \hat{n}_{\downarrow}, \\
\hat{H}_{R} & =\sum_{k, \sigma=\uparrow, \downarrow} \varepsilon_{k, \sigma} \hat{c}_{k, \sigma}^{\dagger} \hat{c}_{k, \sigma}, \\
\hat{H}_{T} & =\sum_{k}\left(\mathcal{T}_{\uparrow}(u) \hat{c}_{k, \uparrow}^{\dagger} \hat{d}_{\uparrow}+\mathcal{T}_{\downarrow} \hat{c}_{k, \downarrow}^{\dagger} \hat{d}_{\downarrow}+\text { H.c. }\right) .
\end{aligned}
$$

The Hamiltonian $\hat{H}_{\mathrm{QD}}$ represents the QD, $\hat{H}_{R}$ the spinpolarized reservoirs (e.g., $\hat{c}_{k, \sigma}$ is an annihilation operator), and $\hat{H}_{T}$ electron tunneling on and off the QD, characterized by the transition integrals $\mathcal{T}_{\sigma}$ between states in the QD and in the reservoirs. The tunneling coupling $\mathcal{T}_{\uparrow}(u)$ varies exponentially with the mechanical deflection $u(t)[19,20]$.

The capacitive backaction force depends on the probability of double occupation $P_{2}=\operatorname{Tr}\left\{\hat{\rho} \hat{n}_{\uparrow} \hat{n}_{\downarrow}\right\}$ and the electronic occupation probabilities $n_{\sigma}=\operatorname{Tr}\left\{\hat{\rho} \hat{n}_{\sigma}\right\}=P_{\sigma}+$ $P_{2}$. To calculate these, we use standard techniques [25-27] to reduce the quantum Liouville equation (3) to rate equations for these probabilities:

$$
\begin{aligned}
& \partial_{t} n_{\uparrow}=-\Gamma_{\uparrow} e^{-\left(u-u^{\mathrm{s}}\right) / \lambda}\left(n_{\uparrow}-f_{1}^{\uparrow}+\Delta f^{\uparrow} n_{\downarrow}\right), \\
& \partial_{t} n_{\downarrow}=-\Gamma_{\downarrow}\left(n_{\downarrow}-f_{1}^{\downarrow}+\Delta f^{\downarrow} n_{\uparrow}\right), \\
& \partial_{t} P_{2}=-\Gamma(u) P_{2}+\Gamma_{\uparrow} e^{-\left(u-u^{\natural}\right) / \lambda} f_{2}^{\uparrow} n_{\downarrow}+\Gamma_{\downarrow} f_{2}^{\downarrow} n_{\uparrow},
\end{aligned}
$$

valid at high temperatures $k_{B} T_{\sigma} \gg \hbar \omega, \hbar \Gamma_{\uparrow}, \hbar \Gamma_{\downarrow}$ (see Supplemental Material [28]). Here, the tunneling rates $\Gamma_{\downarrow}, \Gamma_{\uparrow} \exp \left[-\left(u-u^{\mathrm{st}}\right) / \lambda\right], \Gamma(u)=\Gamma_{\uparrow} \exp \left[-\left(u-u^{\mathrm{st}}\right) / \lambda\right]+$ $\Gamma_{\downarrow}$, where $\lambda$ is an effective tunneling length. The rates are in agreement with Fermi's golden rule and are for convenience defined at the static mechanical displacement $u^{\text {st }}$ which should be found self-consistently (see Supplemental Material [28]). For brevity, we introduced $f_{\alpha}^{\sigma}=f_{F}\left[E_{\alpha}^{\sigma}(u) /\left(k_{B} T_{\sigma}\right)\right]$, where $f_{F}$ is the Fermi distribution function and $\Delta f^{\sigma}=f_{1}^{\sigma}-f_{2}^{\sigma}$. Without an electron-electron interaction, $\Delta f^{\sigma}=0$ and the spin populations decouple. Each occupation probability $n_{\sigma}$ then simply decays towards equilibrium with its corresponding reservoir. A nonzero electron-electron interaction couples the two spin populations.

The mechanical deflection affects the electron dynamics by changing (i) the tunneling rate between the QD and the tip and (ii) the energies of the QD states. The latter of these effects is capable of generating feedback [5]. However, the first effect is much stronger due to a higher sensitivity to the deflection. Indeed, the tunneling rate is sensitive to deflections on the order of the tunneling length $\lambda$, while the Fermi factors $f_{\alpha}^{\sigma}$ change on the length scale $k_{B} T_{\sigma} C^{2} /\left[f_{\alpha}^{\sigma}\left(1-f_{\alpha}^{\sigma}\right) e^{2} \partial_{u} C\right] \gtrsim k_{B} T_{\sigma} 2 \pi \epsilon_{0} L D / e^{2}$, which, for realistic parameters, is much larger $\left(\epsilon_{0}\right.$ is the vacuum permittivity, $L$ is the length of the CNT, and $D$ is the distance to the gate; capacitance modeled as a wire above a plane). Therefore, we will ignore the slight dependence on $u$ in the Fermi factors $f_{\alpha}^{\sigma}$. In this approximation, the timedependent parts of the general solutions for $n_{\uparrow}(t)$ and $n_{\downarrow}(t)$ are transient, and the solutions for long times are simply the static solutions $n_{\sigma}(t)=n_{\sigma}^{\text {st }}$.

One can immediately see that the two single-electron terms in the capacitive force of Eq. (2) do not change in time and, thus, cannot lead to a mechanical instability. In contrast, however, the probability of double occupation, $P_{2}$, will change in time. It enters into the force (2) via the electron-electron interaction term and leads to a dynamical backaction force that can cause a mechanical instability. The fact that $n_{\uparrow}$ and $n_{\downarrow}$ do not change in time means that the average QD charge $-e\left(n_{\uparrow}+n_{\downarrow}\right)$ is constant. That there can still be a time-dependent capacitive force may seem surprising at first. However, the average of the square of the charge, $e^{2}\left[n_{\uparrow}+n_{\downarrow}+2 P_{2}(t)\right]$, does change in time. Hence, there is a dynamical feedback in the system generated by charge variations on the CNT, although these variations do not affect the average charge at any given time.

To investigate the stability of the mechanical subsystem, one could at this point linearize Eqs. (4) and (1) near their stationary solutions and derive a condition for mechanical 
actuation. However, with the aim to study nonlinear effects, we will instead proceed by deriving a closed equation for the CNT deflection (see Supplemental Material [28]). For dimensionless deflection $x(t) \equiv u(t) / \lambda$, time $\tau \equiv t \omega$, and total tunneling rate $\tilde{\Gamma}(x) \equiv \Gamma(u) / \omega$, the closed equation for the mechanical motion is

$$
\partial_{\tau}^{2} x+\frac{1}{Q} \partial_{\tau} x+x=\mathfrak{F}_{\mathrm{st}}+\varepsilon \mathfrak{F}(\tau)\{x\},
$$

where $\mathfrak{F}_{\text {st }}$ is the static component of the capacitive force, $\mathfrak{F}$ is the dynamic backaction force from the electronic subsystem,

$$
\mathfrak{F}(\tau)\{x\}=\int_{-\infty}^{\tau} d \tau^{\prime} e^{-\int_{\tau^{\prime}}^{\tau} d \tau^{\prime \prime} \tilde{\Gamma}\left[x\left(\tau^{\prime \prime}\right)\right]}\left(e^{-x\left(\tau^{\prime}\right)}-1\right),
$$

which is a functional of the mechanical deflection, and $\varepsilon=\left(-\partial_{u} U\right) W /\left(m_{\mathrm{eff}} \omega^{3} \lambda U\right)$. We see that the backaction force is, as already mentioned, proportional to the static component of the heat flow (see Supplemental Material [28]):

$$
W=U \frac{\Gamma_{\uparrow} \Gamma_{\downarrow}}{\Gamma_{\uparrow}+\Gamma_{\downarrow}} \xi\left(T_{\uparrow}, T_{\downarrow}\right),
$$

where

$$
\xi\left(T_{\uparrow}, T_{\downarrow}\right)=\frac{\frac{1}{2} \sinh \left[\frac{U\left(T_{\uparrow}-T_{\downarrow}\right)^{2} k_{B} T_{\uparrow} T_{\downarrow}}{2}\right]}{4 \prod_{\alpha, \sigma} \cosh \left[\frac{E_{\alpha}^{\sigma}}{2 k_{B} T_{\sigma}}\right]-\prod_{\sigma} \sinh \left[\frac{U}{2 k_{B} T_{\sigma}}\right]} .
$$

As mentioned above, the dynamic backaction force is entirely generated by the variations in the probability to be in the double-populated state, $P_{2}(t)$. Hence, the positions of the single-electron levels affect the force only quantitatively; see Eq. (8).

The electrostatic backaction force pumps the mechanical motion and can lead to a mechanical instability. To see when the instability occurs, we linearize Eq. (5) around the static displacement. By analyzing the Lyapunov exponents of $x(t)$, the criterion for mechanical instability is found to be

$$
\frac{\left(-\partial_{u} U\right)}{m_{\text {eff }}} \omega \lambda \frac{\Gamma_{\uparrow} \Gamma_{\downarrow}}{\Gamma\left(\omega^{2}+\Gamma^{2}\right)} \xi\left(T_{\uparrow}, T_{\downarrow}\right)>\frac{1}{Q},
$$

where $\Gamma=\Gamma_{\uparrow}+\Gamma_{\downarrow}$. The instability threshold is reached when the heat-flow-induced pumping overcomes the intrinsic mechanical damping of the CNT. The suggested actuation mechanism relies on two effects. First, the Coulomb interaction energy responsible for the heat flow depends on the mechanical deflection and thus generates a force on the mechanical motion, i.e., $-\partial_{u} U \neq 0$; see Eq. (9). Second, the mechanical deflection also affects the tunneling and thereby the electron dynamics, establishing a dynamical feedback. This is seen in the condition (9), since if the tunneling is insensitive to the mechanical deflection, $\lambda \rightarrow \infty$, the instability criterion cannot be met. Furthermore, the direction of the feedback force changes with that of the temperature difference; see Eq. (8). This means that the effect of the mechanism can be switched between pumping and damping by reversing the direction of the heat flow, regardless of the direction of the electrostatic force.

To estimate the experimental feasibility of the suggested actuation mechanism, we assume typical parameter values (see Table I) and rates optimal for pumping, $\Gamma_{\uparrow}=\Gamma_{\downarrow}=$ $\omega / 2$. The condition (9) then reduces to $\xi\left(T_{\uparrow}, T_{\downarrow}\right)>10^{3} / Q$. This looks promising from an experimental viewpoint, since the magnitude of $\xi\left(T_{\uparrow}, T_{\downarrow}\right)$ grows with the temperature difference, asymptotically approaching $1 / 4$ when $k_{B}\left|T_{\uparrow}-T_{\downarrow}\right| \gg U$.

When the instability criterion (9) is fulfilled, the CNT begins to vibrate with increasing amplitude. The amplitude is eventually stabilized, since the pumping efficiency decreases with the amplitude. To find the stationary amplitude, we exploit the smallness of $\varepsilon$ (see Table I) and use the Krylov-Bogoliubov averaging method [31] on Eq. (5). It is then assumed that the CNT deflection has the form $x(\tau)=x^{\mathrm{st}}+A(\tau) \cos [\tau+\phi(\tau)]$, where both the amplitude $A(\tau)$ and the phase $\phi(\tau)$ are slowly varying functions. We get an equation for the rate of change of the amplitude:

$\partial_{\tau} A=-\frac{A}{2 Q}-\frac{\varepsilon}{2 \pi} \int_{0}^{2 \pi} d \tau^{\prime} \sin \left(\tau^{\prime}\right) \mathfrak{F}\left(\tau^{\prime}\right)\left\{A \cos \left(\tau^{\prime \prime}\right)\right\}$.

A stable oscillation is reached when the backaction pumping is balanced by the mechanical damping. Numerical calculations of the pumping term in Eq. (10) are presented in Fig. 3 (see the figure caption for the parameters). We see that the amplitude of the oscillations stabilizes at a value on the order of $\lambda$, the tunneling length.

We estimate the heat-engine efficiency of the system at these parameters to less than one percent for $k_{B} T_{\uparrow}>U$. If the quality factor is increased to $Q=10^{5}$, we reach efficiencies of a few percent. When $k_{B} T_{\uparrow} \gg U \gg k_{B} T_{\downarrow}$, we find that the temperature dependence of the efficiency becomes negligible and it becomes dominated by geometrical factors.

We have proposed an electromechanical instability in a system without an exchange of electrons between the reservoirs. In the presented prototype system, the electronic current through the device was blocked by a spin-valve effect. However, spin polarization is not a requirement. Another way to prevent the charge current would be to use two spatially separated two-level QDs. Tunneling between the QDs can then be suppressed while the electron-electron interaction remains significant.

TABLE I. Typical parameter values [29,30].

\begin{tabular}{lc}
\hline \hline$m_{\mathrm{eff}} \sim 10^{-21} \mathrm{~kg}$ & $U \sim e^{2} / C \sim 10^{-4} \mathrm{eV}$ \\
$\lambda \sim 0.1 \mathrm{~nm}$ & $\omega \sim 1 \mathrm{GHz}$ \\
$D \sim 100 \mathrm{~nm}$ & $\varepsilon \sim 10^{-2} \ll 1$ \\
$L \sim 1 \mu \mathrm{m}$ & \\
\hline \hline
\end{tabular}




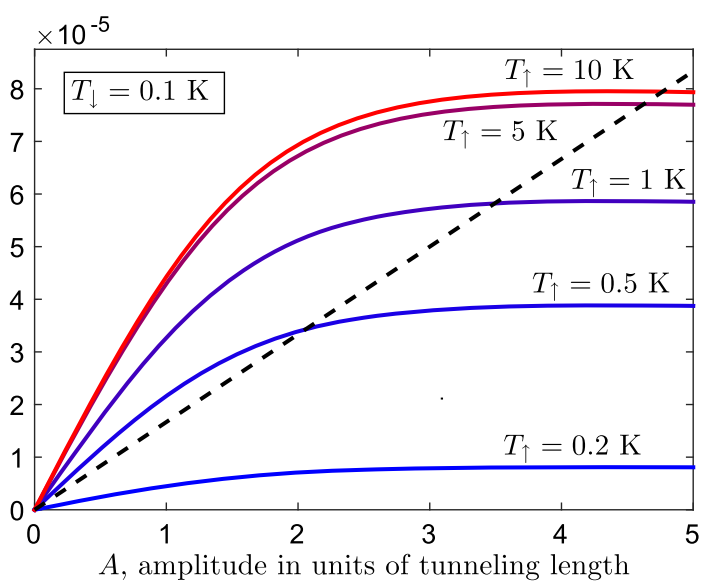

FIG. 3. The intrinsic mechanical damping (dashed line) and heat-flow-induced pumping (solid lines), given by the first and second term, respectively, in the rhs of Eq. (10). If the pumping overcomes the damping, the system is actuated to an amplitude where the two are balanced (line intersections). We use parameters as in Table I and $\Gamma_{\sigma}=\omega / 2, E_{1}^{\sigma}=-U / 2(\sigma=\uparrow, \downarrow)$, which are the optimal values for pumping, and $Q=3 \times 10^{4}$. The pumping is shown for different temperatures $T_{\uparrow}$ of the hot reservoir, while the cold reservoir is kept at $T_{\downarrow}=0.1 \mathrm{~K}$.

The mechanical vibrations could be detected by, e.g., applying a magnetic field perpendicular to the CNT and its motion. A charge residing on the CNT would then experience a Lorentz force which alternates with the deflection. The mechanical vibrations can then be read out by measurement of the resulting alternating current between the two leads. Note that the current does not flow between the reservoirs referred to in our system, since the two leads together constitute a single reservoir.

In conclusion, we have shown that a nanoelectromechanical system can be actuated by a heat flow using a positiondependent electron-electron interaction. This actuation mechanism differs from many others in that it does not involve any charge or spin current nor any external ac fields. The amplitude of the actuated oscillations is stabilized, since the pumping efficiency decreases with the amplitude. Our estimations show that the considered phenomena can be studied using existing experimental techniques.

We thank the Swedish Research Council (VR) for funding.

*Corresponding author. anton.vikstrom@chalmers.se

[1] K. L. Ekinci and M. L. Roukes, Rev. Sci. Instrum. 76, 061101 (2005).

[2] B. Lassagne, Y. Tarakanov, J. Kinaret, D. Garcia-Sanchez, and A. Bachtold, Science 325, 1107 (2009).

[3] S. Sapmaz, Y. M. Blanter, L. Gurevich, and H. S. J. van der Zant, Phys. Rev. B 67, 235414 (2003).
[4] A. K. Hüttel, B. Witkamp, M. Leijnse, M. R. Wegewijs, and H. S. J. van der Zant, Phys. Rev. Lett. 102, 225501 (2009).

[5] Y. M. Blanter, O. Usmani, and Y. V. Nazarov, Phys. Rev. Lett. 93, 136802 (2004).

[6] F. Pistolesi and R. Fazio, New J. Phys. 8, 113 (2006).

[7] F. Santandrea, L. Y. Gorelik, R. I. Shekhter, and M. Jonson, Phys. Rev. Lett. 106, 186803 (2011).

[8] P. Stadler, W. Belzig, and G. Rastelli, Phys. Rev. Lett. 113, 047201 (2014).

[9] J. Atalaya and L. Y. Gorelik, Phys. Rev. B 85, 245309 (2012).

[10] K. Ekinci, Small 1, 786 (2005).

[11] W.-M. Zhang, H. Yan, Z.-K. Peng, and G. Meng, Sens. Actuators A 214, 187 (2014).

[12] E. M. Miandoab, A. Yousefi-Koma, H. N. Pishkenari, and F. Tajaddodianfar, Commun. Nonlinear Sci. Numer. Simul. 22, 611 (2015).

[13] F. Tajaddodianfar, H. N. Pishkenari, M. R. H. Yazdi, and E. M. Miandoab, Commun. Nonlinear Sci. Numer. Simul. 20, 1078 (2015).

[14] D. Garcia-Sanchez, A. M. van der Zande, A. S. Paulo, B. Lassagne, P. L. McEuen, and A. Bachtold, Nano Lett. 8, 1399 (2008).

[15] Y. Xu, C. Chen, V. V. Deshpande, F. A. DiRenno, A. Gondarenko, D. B. Heinz, S. Liu, P. Kim, and J. Hone, Appl. Phys. Lett. 97, 243111 (2010).

[16] Q. P. Unterreithmeier, E. M. Weig, and J. P. Kotthaus, Nature (London) 458, 1001 (2009).

[17] K. R. Brown, J. Britton, R. J. Epstein, J. Chiaverini, D. Leibfried, and D. J. Wineland, Phys. Rev. Lett. 99, 137205 (2007).

[18] T. J. Kippenberg and K. J. Vahala, Science 321, 1172 (2008).

[19] L. Y. Gorelik, A. Isacsson, M. V. Voinova, B. Kasemo, R. I. Shekhter, and M. Jonson, Phys. Rev. Lett. 80, 4526 (1998).

[20] A. Isacsson, L. Y. Gorelik, M. V. Voinova, B. Kasemo, R. I. Shekhter, and M. Jonson, Physica (Amsterdam) 255B, 150 (1998).

[21] T. Novotný, A. Donarini, and A.-P. Jauho, Phys. Rev. Lett. 90, 256801 (2003).

[22] P. Stadler, W. Belzig, and G. Rastelli, Phys. Rev. Lett. 117, 197202 (2016).

[23] B. J. LeRoy, S. G. Lemay, J. Kong, and C. Dekker, Nature (London) 432, 371 (2004).

[24] M. Ziese, Rep. Prog. Phys. 65, 143 (2002).

[25] H. Breuer and F. Petruccione, The Theory of Open Quantum Systems (Oxford University, New York, 2002).

[26] L. Y. Gorelik, D. Fedorets, R. I. Shekhter, and M. Jonson, New J. Phys. 7, 242 (2005).

[27] G. Schaller, Open Quantum Systems Far from Equilibrium, Lecture Notes in Physics (Springer, New York, 2014).

[28] See Supplemental Material at http://link.aps.org/ supplemental/10.1103/PhysRevLett.117.247701 for the derivation of the electronic master equation, the closed equation for the mechanical motion, and the static solution.

[29] A. Benyamini, A. Hamo, S. V. Kusminskiy, F. von Oppen, and S. Ilani, Nat. Phys. 10, 151 (2014).

[30] J. Chaste, M. Sledzinska, M. Zdrojek, J. Moser, and A. Bachtold, Appl. Phys. Lett. 99, 213502 (2011).

[31] N. M. Krylov and N. N. Bogoliubov, Introduction to Nonlinear Mechanics (Princeton University, Princeton, NJ, 1949). 